\title{
Extraction of road congestion information by change detection of multi-temporal satellite observing images
}

\author{
Yan Wang ${ }^{1}$, Hong Zhou ${ }^{2}$, Hua Xu ${ }^{3,4}$, Zhonghua He ${ }^{4, a}$, and Liping Lei ${ }^{4}$ \\ ${ }^{1}$ Highway Monitoring and Response center, Ministry of Transport, China \\ ${ }^{2}$ Jiangsu Communications Holding Co., Ltd, China \\ ${ }^{3}$ School of Earth Sciences and Resources, China University of Geosciences (Beijing), China \\ ${ }^{4}$ Institue of Remote Sensing and Digital Earth, Chines Academy of Science, China
}

\begin{abstract}
Road congestion has been significantly affecting our live and environment. It is important to get the information of traffic congestion in a real time. In this study, we present an approach by the detecting the vehicles to view the traffic conditions using the satellite observing image from Gaofen-4 (GF-4). GF-4 is a fixed satellite which obtains the images with a high timely interval of $20 \mathrm{~s}$ but its spatial resolution is $50 \mathrm{~m}$. This study seeks the application of this satellite observation to get the information of traffic congestion using its advantage of high time-frequency observation by the change detection of images over the road when the different number of vehicle are occupying over the road. We used the high spatial resolution (1m) images of Gaofen-2 (GF-2) to simulate the scenario of different road congestions, after that the simulated images is transferred into Gaofen- 4 data by down the spatial resolution to $50 \mathrm{~m}$. The result of our study shows that heavy road congestion over the highway, the length of road congestion is larger than $1 \mathrm{~km}$, can be detected from 50m GF-4 images. The result demonstrates that the observation of GF-4 could be an alternate way for the real time monitoring of traffic condition.
\end{abstract}

Keywords: road congestion; change detection; satellite observation; multi-temporal.

\section{Introduction}

Road congestion has been significantly affecting our live and environment in many countries like China with high-speed development of the road. Real-time traffic information is needed by the civilian for their routes at this information era. And comprehensive, reliable and timely information of traffic status is also useful for the plan of the traffic warden.

Current monitoring equipments are not efficiency enough to the large range road congestion [1]. Induction loops, overhead sensors, video system and other timely detection equipment are installed in the current traffic systems [2]. But the coverage area of their detection is limited. However, with the cost restriction and influence of safety factors, it is difficult to increase the quantity of this equipment rapidly. And the cost management, maintenance of equipment and information accuracy influenced by the local environment are important problems for the continuous real time inspection of these traditional detections. Moreover it is problem for collaborative analysis and application of different forms from various detection equipment. .The technology of remote sensing is known as an alternate

\footnotetext{
${ }^{\mathrm{a}}$ Corresponding author : hezhonghua1992@163.com
} 
way to monitoring the road congestion as it is not only stable observation monitoring but also relatively low cost.

Images from airborne sensors or satellite observations have been used in vehicle detection and traffic status monitoring [3-4]. Although, some approaches based on aerial imagery are sophisticated and deliver good results, its high cost for multi-temporal and high spatial coverage limit its timely monitoring. In addition, images with resolution better than $1 \mathrm{~m}$ have been widely used in road or vehicle detection [5-6]. And results show that using high resolution satellite images for road status detection is an efficient way for urban transportation planning [4]. And they can give users instantaneous traffic status for their imaging time. But their time resolution is too low to conduct the timely monitoring.

With the technology development of remote sensing and information, more high resolution with multi-temporal remote sensing satellite data products are going to be available [7]. In this study, we propose an approach by the detecting the vehicles to view the traffic conditions using the satellite observing image from GF-2 and GF-4.

GF-2 is the first national civil optical remote sensing satellite with five bands, developed by China. And the spatial resolution of its panchromatic (PAN) band is superior to $1 \mathrm{~m}$. It was launched successfully on August 19, 2014, and starting up to image and transmit data two days later. And GF-2 was mainly used in the government sectors, including Ministry of Land and Resources, Ministry of Housing and Urban-Rural Development of the People's Republic of China, Ministry of Transport of the PRC, State Forestry Administration and other departments. GF-4 is the first geosynchronous orbit satellite in China with spatial resolution of $50 \mathrm{~m}$ and time resolution of $20 \mathrm{~s}$ except for the medium wave infrared band with spatial resolution of $400 \mathrm{~m}$. It was successfully launched on December 29, 2015, and images of real observations are in check. The PAN of GF-2 and GF-4 is shown in table 1. And more information about the parameters of GF-2 and GF-4 can be achieved from the website of China Centre For Resources Satellite Data and Application (http://www.cresda.com/EN/index.shtml).

GF-2 imagery has been efficiently used for scientific research, like assistance for urban forest mapping [8]. And expanding the application of GF-2, a high spatial resolution satellite, is meaningful. Besides, GF-4 has its advantage of multi-temporal imaging with time resolution of $20 \mathrm{~s}$ that will be useful for timely monitoring. Considering current problem of no reliable method for timely detecting traffic status with high coverage, GF-4 imagery could be a candidate dataset for helping solving it. Even though, the images of GF-4 is not available at present, verifying the availability of GF-4 applying the road congestion monitoring is valuable for real time monitoring of road congestion from satellite observations. And we conduct this research with the method of data simulation, change detection and statistical method for extracting information of road congestion. Considering lack of GF-4 dataset, parameters of GF-2 and GF-4 was compared in table 1.

Table 1. Information of panchromatic (PAN) about Gaofen-2 and Gaofen-4.

\begin{tabular}{|c|c|c|c|c|c|c|c|}
\hline & $\begin{array}{c}\text { spectral } \\
\text { range }(\mu \mathrm{m})\end{array}$ & Orbit Type & $\begin{array}{c}\text { Repetition } \\
\text { cycle }\end{array}$ & $\begin{array}{c}\text { Spatial } \\
\text { resolution }\end{array}$ & $\begin{array}{c}\text { Swath } \\
\text { width }\end{array}$ & $\begin{array}{c}\text { Fix point } \\
\text { location }\end{array}$ & local-time \\
\hline GF-2 & $0.45 ~ 0.90$ & Sun-synchronous & 5 days & $0.81 \mathrm{~m}$ & $45 \mathrm{~km}$ & ----- & $10: 30 \mathrm{AM}$ \\
\hline GF-4 & $0.45 \sim 0.90$ & Geosynchronous & $20 \mathrm{~s}$ & $50 \mathrm{~m}$ & $400 \mathrm{~km}$ & $105.6^{\circ} \mathrm{E}$ & ----- \\
\hline
\end{tabular}

\section{Methodology}

\subsection{Study area and data}

\subsubsection{Study area}

The study area is a section of G6 Jingzang Expressway located in the north-eastern of Haidian district, Beijing, China (Figure 1). It is one arterial road connecting the Beijing city and the suburbs. For the 
period of usual rush hours and important dates, like the National People's Congress and the Chinese Political Consultative Conference (NPC \& CPPCC), the traffic pressure there is tremendous. Around the study area, building land is the main cover type, including residential area, schools, and business office district. People are easily gathered with cars increase, and then the road congestion occurs. For confirming the usability of GF-4 in road congestion and verification later, we select images there for the experiment.

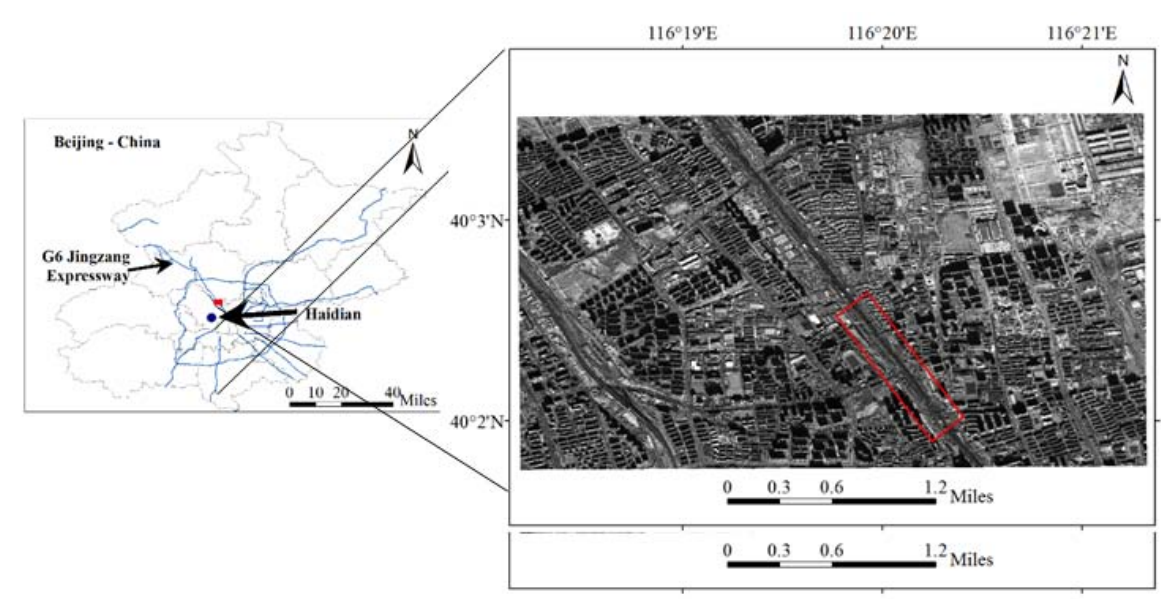

Figure 1. Location of this study area. Blue lines in the left is the main high ways around Beijing. And the red box left is the area of GF-2 data shown in the right. Red rectangle right shows the region of interest (ROI) in G6 Jingzang Expressway for road congestion monitoring with multi-temporal images.

\subsubsection{Data collection}

Cloud-free GF-2 data, the background image, were acquired on 5 December 2014, covering the total of ROI. And multi-temporal data is simulated from the background image. Vehicles can be obliviously identified. Some of them were extracted by us to cover in the background image, and the different traffic status imagery can be gotten. According to the Table 1, the PAN spectral range of GF-2 and GF-4 is same with each other. Besides, they are the same series satellite with some similar sensor device. So we attempt to simulate multi-temporal GF-4 images (SGF-4) by images of GF-2. In order to add some data error for different viewing conditions, different rules and multiple process resampling and is adopted.

\subsection{Simulation for the different road congestion scenarios and change detection}

Image of GF-2 T2 was simulated by change the number and distribution of vehicles in GF-2 T1 image. And the images of GF-2 T1, T2 were used to simulate the corresponding images of SGF-4 by resampling process. Then, the process of geometrical rectification and normalization is conducted in these different temporal images. Combining with geographic data of road file, road information of SGF-4 can be extracted for change extraction. And the change ratio can be gained. For selecting areas of road congestion, a threshold with ratio of 0.05 is chosen for the judgement in the study, which is selected by the visual interpretation and result of statistical analysis. After getting the change information, we did the post-processing with expert knowledge and road file for get more precise traffic information. The main process of this technology is shown in figure 2.

Visual interpretation was used for vehicle extraction and the tool of Adobe Photoshop CS4 was applied to get different road status images. Multiple times resampling and different start line were adopted for simulating GF-4 images based on GF-2 data. The road file and tool of extract by mask in 
ARCGIS 10.1 were used for road information extraction. And then, the Ratio (l, $\left.\mathrm{t}_{2}\right)$ from equation (1) was used as evaluation index in change detection.

$$
\text { Ratio }(1, t 2)=(G F-4(1, t 2)-G F-4(1, t 1)) /(G F-4(1, t 2)+G F-4(1, t 1))
$$

Ratio $\left(I, t_{2}\right)$ indicates the change degree of road status in time of $t_{2}$ compared with it in time of $t_{1}$. ' $P$ ' represent the location of change detection area and ' $t_{1}$ ' and ' $t_{2}$ ' represent the time of $t_{1}$ and $t_{2}$. And $G F-4\left(l, t_{j}\right)$ represent data of GF-4 image in location of $l$ and time of $\mathrm{t}_{\mathrm{i}}$.

Statistic result of Ratio $\left(l, t_{2}\right)$ distribution and visual judgement were used for choosing the threshold, and the result in this study was identified as 0.05 . According to the prior knowledge that when road is in congestion, the distance between adjacent car group is less than $200 \mathrm{~m}$ and the length of congestion line is more than $1 \mathrm{~km}$ at usual. We use them as a criteria to identify status of road congestion for computer programing of information extraction automatic processing.

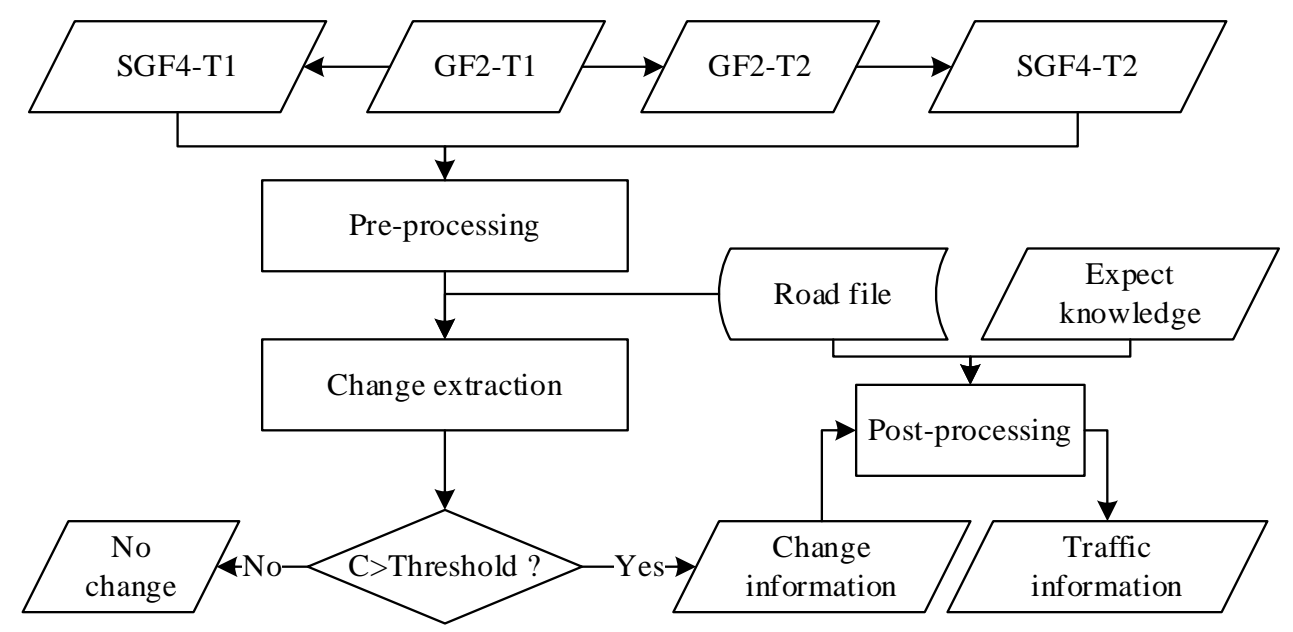

Figure 2. The main technological process in this study.

\section{Results and discussions}

The experiment results are based on GF-2 and SGF-4 image, a section of G6 Jingzang Expressway in the north-eastern of Haidian District, Beijing, China. Concentrate on the ROI, different traffic status images can be simulated by the background data, GF-2 image, and vehicle information inside. And we adopted three kinds of road congestion status images including background, mild-congestion and heavy congestion on the road. Corresponding quantity of cars on the road are less than 100, around 200 and more than 400. Dataset and extract results will be shown below.

\subsection{Dataset of different traffic status in GF-2}

The road status can be distinguished from GF-2 with method of visual interpretation by zoomed image. And the result is shown in figure 3 that (a) is image of no congestion, (b) represent mild congestion and (c) shows the heavy congestion. The bottom three images are the zoomed images of red box in the top images in figure 3. For no congestion image, sparse vehicles lie in the high way. And we can recognize it as smooth traffic obviously. However, Start from the entrance ramp in the image, length of road congestion is around 280 meters shown in image of mid-congestion. Furthermore, road congestion length in heavy-congestion status is up to 1000 meters that can be measured from the image right. And the red lines below of (b) and (c) in figure 3 show the section of road congestion. 


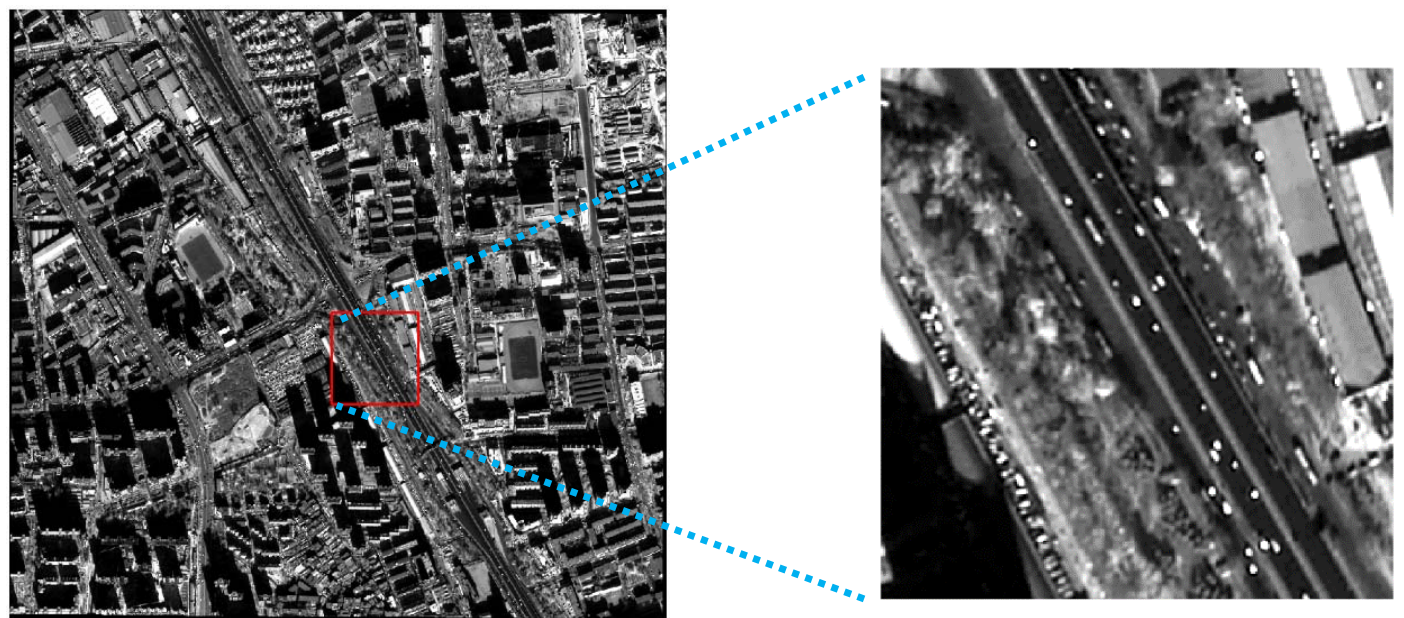

(a) no-congestion

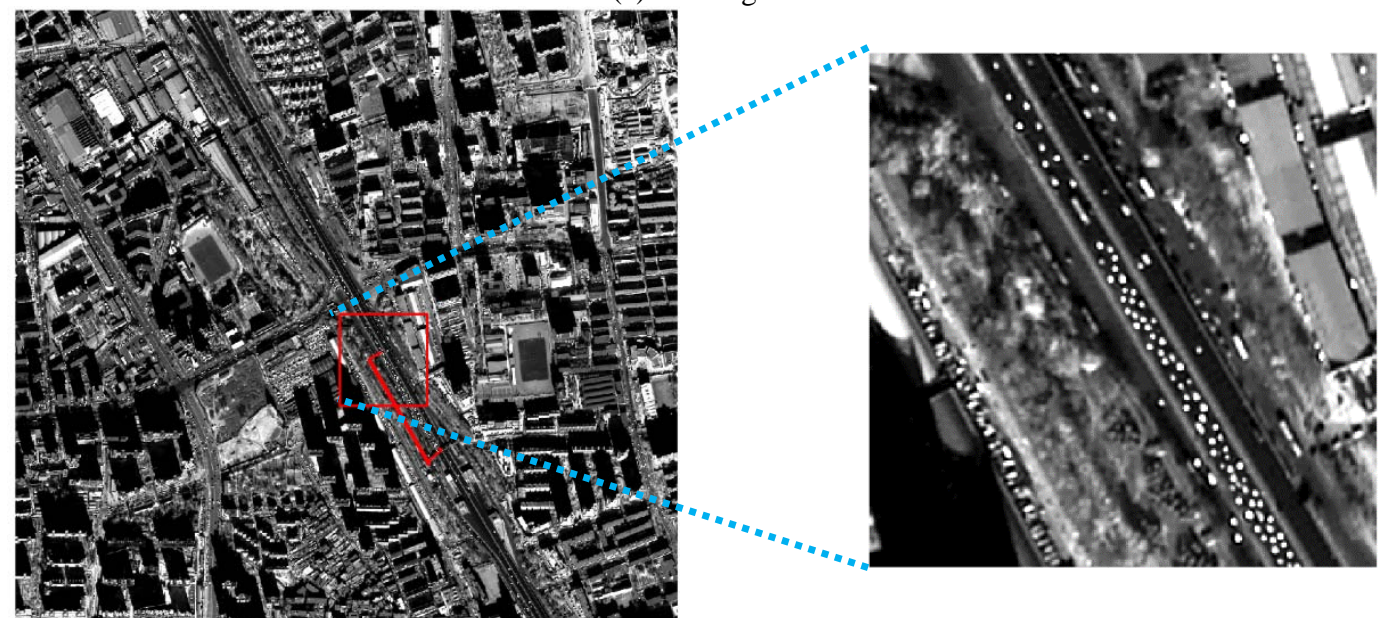

(b) mild-congestion

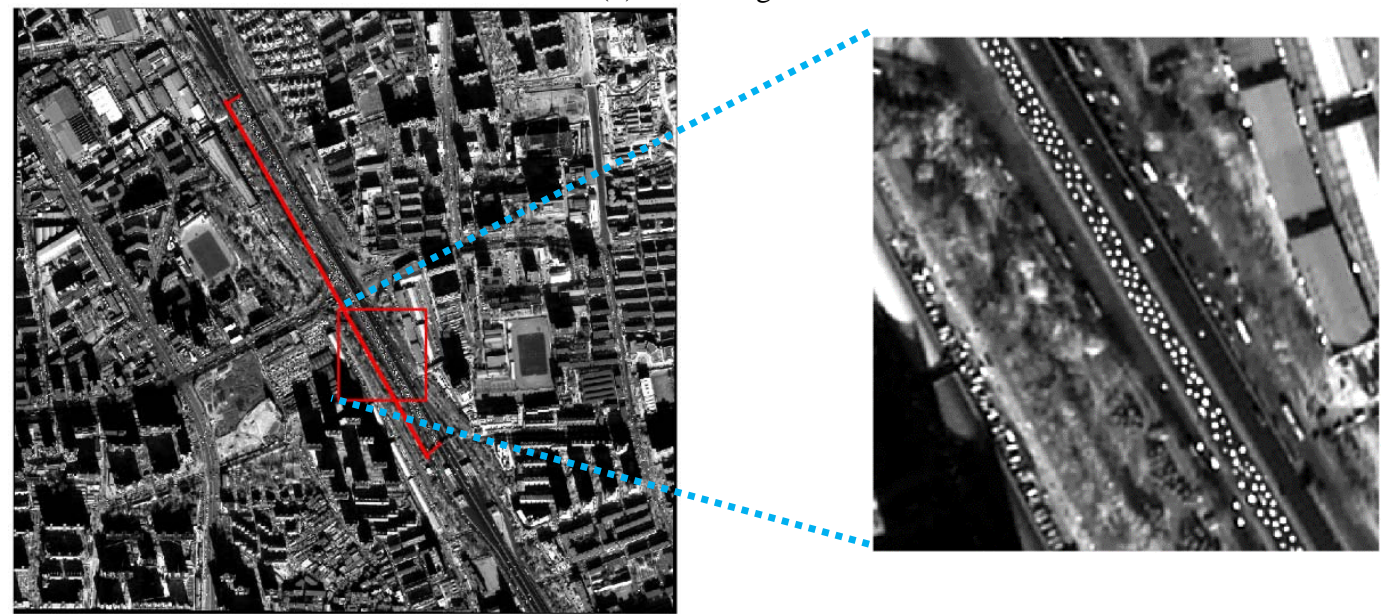

(c) heavy-congestion

Figure 3. Simulated scenarios of the different traffic congestion in GF-2 over $1 \mathrm{~km}$ long road with (a) no-congestion less than 100 cars, (b) mid congestion about 200 cars and (c) heavy congestion more than 400 cars on the target highway as shown in Figure 1. 


\subsection{Different traffic status in SGF-4 imagery}

The multi-temporal images of SGF-4 are simulated by GF-2 image that are shown in figure 3. With the spatial resolution of $50 \mathrm{~m}$, single vehicle information can't be recognized. But the broad outlines and significant different surface reflectance and DN value exist. Under the ideal conditions, this different can be used for change extraction. For the serious road congestion areas, the DN value is very high. Besides, the road congestion section interpreted from GF-2 is shown with red line in the figure 4.

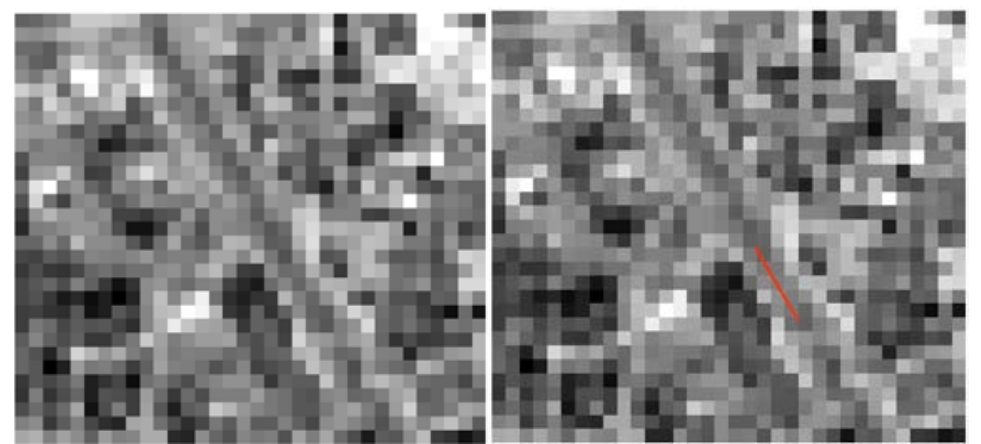

(a) no-congestion (b) mild-congestion

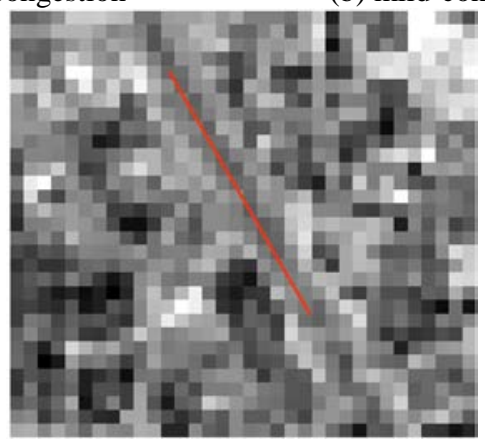

(c) heavy-congestion

Figure 4. Different traffic status in SGF-4 with less than 100 cars in no-congestion, around 200 in the mid congestion and more than 400 in heavy congestion on the target street shown in figure 1 . And the red line in (b) and (c) indicate the serious road congestion section interpreted from figure 3.

\subsection{Extracted results}

The equation (1) was used in calculating the different ratio of change extraction. And the results contain ROI were achieved as shown in figure 5. Concentrating on the highway areas, high ratio was extracted and shown with red grid which indicates road congestion section. Compared with road congestion information from GF-2, the main serious traffic road sections can be extracted by the SGF-4 images no matter with mild or heavy congestion. And the heavy-congested road sections can be better detected. For that, using multi-temporal images from satellite to extract serious road congestion information in the highway is possible. However, this is an exploratory work with simulated data and basic statistical method for the ideal condition. More work is needed for eliminating the image error, lessening statistical uncertainty and increasing the applicability of objective but variable threshold. 


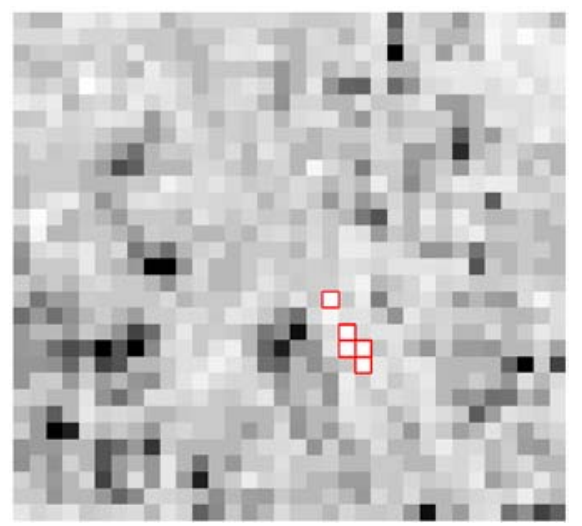

(a) Extraction result of mild-congestion

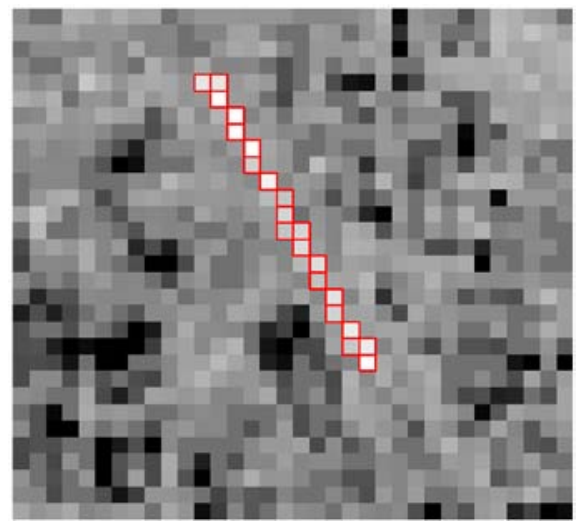

(b) Extraction result heavy-congestion

Figure 5. The extraction result of different road congestion. Red grids show high ratio that lager than the threshold (0.05 here) in the G6 Jingzang Expressway which indicate the result of congestion extraction.

\section{Conclusions}

We developed an approach to extract the traffic congestion information by detecting the vehicle over the road using GF-4 with the high time-frequency observations. The results show that this approach is valid for the condition of heavy road congestion over the highway, and could be potentially applied to the near real-time monitoring of road congestion. However, it is important that the quality of satellite observing image and speed of data processing for guaranteeing the availability of this method. For that, GF-4 images from the multi-temporal observation should be normalized before applied to this change detecting approach. Moreover, it is limited from the cloud cover which will reduce the timely advantage of GF-4. In future, we need to certify this method using the real GF4 images replacing the simulating images.

\section{Acknowledgement}

The study was supported by the project of "Grand Special of High resolution On Earth Observation: Application demonstration system of high resolution remote sensing and transportation”, Grant NO: 07-Y30B10-9001-14/16.

\section{References}

1. P.-T. Chen, F. Chen, and Z. Qian, IEEE International Conference on Data Mining, Road traffic congestion monitoring in social media with hinge-loss Markov random fields, 80-89 (2014)

2. J. Leitloff, S. Hinz, and U. Stilla, IEEE transactions on Geoscience and remote sensing, Vehicle detection in very high resolution satellite images of city areas, 48, 2795-2806 (2010)

3. K.-H. Bethke, S. Baumgartner, and M. Gabele, Airborne road traffic monitoring with radar (2007)

4. S. O. Larsen, H. Koren, and R. Solberg, Photogrammetric Engineering and Remote Sensing, Traffic Monitoring Using Very High Resolution Satellite Imagery, 75 (2009)

5. F. Gao, B. Li, Q. Xu, and C. Zhong, Remote Sensing, Moving Vehicle Information Extraction from Single-Pass WorldView-2 Imagery Based on ERGAS-SNS Analysis, 6, 6500-6523 (2014)

6. J. W. Gu, Y. Zou, J. Zhang, Y. Zhu, F. Chen, W. Gu, et al., MATEC Web of Conferences, Solutions to Traffic Jam on East Road of Beijing Jiaotong University in Rush Hours Based on Analogue Simulation, 25, 04004 (2015) 
7. S. Baumgartner and G. Krieger, PositionIT-The Geo-Informatics, Surveying, GIS, GPS and Location Based Services Journal for Southern Africa, Traffic monitoring via satellite, 57-62 (2013)

8. H. Wang, C. Wang, and $\mathrm{H}$. Wu, Remote Sensing Letters, Using GF-2 Imagery and the Conditional Random Field Model for Urban Forest Cover Mapping, 7, 378-387 (2016) 